\title{
Processo de enfermagem informatizado em unidade de terapia intensiva: uma prática educativa com enfermeiros
}

\section{Computerized nursing process in intensive therapy unit: an educative practice with the nurses}

\section{Proceso de enfermería informatizado en unidad de terapia intensiva: una práctica educativa con enfermeros}

\author{
Daniela Couto Carvalho Barra', Grace Teresinha Marcon Dal Sasso", Marisa Monticelli"I \\ 'Mestranda pelo Programa de Pós-Graduação em Enfermagem da Universidade Federal de Santa Catarina (PEN/UFSC). Bolsista CNPq .Email: \\ danyccbarra@yahoo.com.br. \\ "Doutora em Informática em Saúde e Enfermagem, Docente do Departamento de Enfermagem e do PEN/UFSC. Email: grace@nfr.ufsc.br. \\ I"' Doutora em Enfermagem; Docente do Departamento de Enfermagem e do PEN/UFSC. Email: marisa@nfr.ufsc.br.
}

\section{RESUMO}

Compreendendo-se que o processo de enfermagem é uma ferramenta imprescindível em Unidades de Terapia Intensiva (UTI), esta pesquisa convergente-assistencial objetivou avaliar a aplicação do Processo de Enfermagem informatizado, a partir da Classificação Internacional das Práticas de Enfermagem - CIPE ${ }^{\circledR}$ versão 1.0 , com os enfermeiros da UTI do Hospital Universitário da Universidade Federal de Santa Catarina. Como estratégia de coleta de dados realizou-se três encontros com cinco enfermeiros intensivistas do hospital. Nos encontros foi utilizado o Arco da Problematização de Maguerez com o suporte teórico da Pedagogia Problematizadora. O estudo foi desenvolvido no período de outubro a novembro de 2007 e os resultados evidenciaram que o processo de enfermagem informatizado em terapia intensiva de acordo com a $\mathrm{CIPE}{ }^{\circledR}$ possui critérios de ergonomia, usabilidade e conteúdo conforme os padrões internacionais, e contribui para a melhoria da prática de enfermagem. Enquanto terminologia de referência para a profissão, os enfermeiros avaliam que esta classificação apresenta linguagem de fácil entendimento, possui aderência com a realidade em foco e, com isso, permite o desenvolvimento da prática de enfermagem baseada na evidência em terapia intensiva.

Descritores: Processos de Enfermagem; Informática em enfermagem; Unidades de terapia intensiva; Classificação; Educação em enfermagem

\section{ABSTRACT}

Understanding that the nursing process is a crucial tool in Intensive Care Units (ICU), this convergent-care research aimed to evaluate the application of the computerized Nursing Process, from the International Classification of Nursing Practices $\left(\right.$ ICNP $^{\circledR}$ ) version 1.0 with the nurses from the Intensive Care Unit of the University Hospital in Santa Catarina. As strategy for data collection three meetings were carried out with five intensive care nurses of the hospital. For the implementation of the meetings the Arc of the Problematical by Maguerez was used with the theoretical support of the Problematical Pedagogy. The study was performed from October to November of 2007 and the results showed that the computerized nursing process in intensive care in according to the ICNP ${ }^{\circledR}$ has criteria of ergonomics, usability and content in accordance with international standards. It also contributes to the improvement of nursing practice. While a terminology of reference for nursing, in agreement with the nurses, this classification showed to be a language of easy understanding and close to the reality and, therefore, allows the development of evidence-based nursing practice in intensive unit.

Descriptors: Nursing process; Nursing informatics; Intensive Care Units; Classification; Education Nursing.

\section{RESUMEN}

Entendiendo que el proceso de enfermería es una herramienta imprescindible en la Unidad de Cuidados Intensivos (UCl), esta investigación convergente asistencial tuvo como objeto evaluar la aplicación del Proceso de Enfermería informatizado, a partir de la Clasificación Internacional para la Práctica de Enfermería - CIPE ${ }^{\circledR}$ versión 1.0 , con los enfermeros de la UCl del Hospital Universitario de la Universidad Federal de Santa Catarina. Para la recolección de los datos se optó por la realización de tres encuentros con cinco enfermeros intensivistas del hospital, utilizando como estrategia de acción el Arco de la Problematización, y como base teórica, la Pedagogía Problematizadora. El estudio fue realizado en el período de octubre a noviembre de 2007 y los resultados mostraron que lo proceso informatizado de enfermería a partir de la CIPE ${ }^{\circledR}$ posee los criterios de ergonomía, usabilidad y contenido conforme los padrones internacionales y contribuye para la mejoría de la práctica de enfermería. Esa clasificación como terminología de referencia para la enfermería, demostró ser una lenguaje de fácil comprensión y próximo de la realidad de la práctica, además, permitiendo así el desarrollo de la práctica de enfermería en terapia intensiva basada en la evidencia.

Descriptores: Procesos de Enfermería; Enfermería informática; Unidades de Terapia Intensiva; Clasificación; Educación en Enfermería. 


\section{NTRODUÇÃO}

Os avanços promovidos pela industrialização trouxeram consigo modernização, além de desenvolvimento tecnológico e científico. Estes avanços ocorreram em todas as áreas de conhecimento e, especificamente na saúde, a tecnologia da informática, dos produtos e dos processos colaborou para a rapidez diagnóstica e o controle das formas de tratamento e de cuidado. Paralelamente, a utilização da tecnologia tem contribuído de forma crescente para a solução de problemas de saúde que antes eram difíceis de serem solucionados com rapidez e segurança. Em razão disso, atualmente, muitas situações graves de saúde, assim como alguns riscos de doenças, estão sendo revertidos em melhores condições de vida e saúde para as pessoas ${ }^{(1-2)}$.

Esta evolução tecnológica e científica exige dos enfermeiros o desenvolvimento de trabalho competente, de complexidade crescente, com habilidades para a tomada de decisão segura, livre de risco e nas mais variadas situações clínicas. Também é imprescindível que este trabalho esteja ancorado na evidência científica. As exigências do processo de cuidar na Unidade de Terapia Intensiva (UTI) determinam que os enfermeiros possuam uma ampla base de conhecimentos científicos e de especializações e que integrem suas habilidades técnicas, intelectuais e de acolhimento à sua prática assistencial e administrativa diária. Neste contexto, as principais funções a serem desempenhadas pelo enfermeiro envolvem procedimentos de avaliação diagnóstica, elaboração de evolução clínica a cada turno de trabalho, exercício de cuidados considerados complexos, participação na realização de procedimentos técnicos altamente especializados, bem como manuseio dos equipamentos disponíveis no cenário de cuidados intensivos ${ }^{(3)}$.

Entende-se que o Processo de Enfermagem (PE) é fundamental em uma UTI, pois, além de integrar e organizar o trabalho da equipe de Enfermagem, diminuindo a fragmentação dos cuidados, garante a continuidade dos mesmos, permitindo tanto avaliar a sua eficácia ou modificá-los, de acordo com os resultados na recuperação do cliente, como também servir de fundamentação permanente para a educação, a pesquisa e o gerenciamento em Enfermagem. Este pressuposto é válido para ambas as formas de implementação do $\mathrm{PE}$, seja para a modalidade tradicional, ou ainda, para a eletrônica. Contudo, diante da constatação de que o registro eletrônico, na área da saúde, vem crescendo no mundo todo(4), ressalta-se aqui essa mesma importância, sob o ponto de vista da enfermagem. Assim, tem-se procurado redobrar esforços no sentido de inserir o Processo de Enfermagem informatizado, integrado ao Prontuário Eletrônico do Paciente.

Entretanto, embora o PE seja considerado por muitos estudiosos da área como um espaço lógico e científico, revestindo-se num consolidado de informação e conhecimento acerca do cuidado de Enfermagem, além de estar legalmente respaldado pelo Conselho Federal de Enfermagem, percebe-se a dificuldade dos enfermeiros de incorporá-lo na prática. Tal fato pode estar relacionado a fatores como: pouco preparo dos profissionais, acúmulo de atividades nos ambientes de cuidado, resistências quanto ao uso da informação estruturada eletronicamente, falta de tempo ou, ainda, porque estes profissionais ainda não perceberam, na prática, o impacto da utilização do PE informatizado ${ }^{(4-6)}$. Em muitos serviços de saúde o trabalho desenvolvido pelos enfermeiros tem sido registrado nos sistemas informatizados apenas como procedimentos médicos para fins de administração e gerenciamento dos recursos.

Sabe-se que as informações em saúde são heterogêneas, distribuídas, complexas e pouco estruturadas. Porém, são centrais no processo de cuidar em saúde. Desta forma, a informática em Enfermagem, enquanto ciência da informação que explicita uma estrutura lógica de dados, informação e conhecimento, tem sido concebida como um caminho para o desenvolvimento do PE e tem contribuído para a melhoria da saúde das pessoas em todo o mundo(7)

A introdução do PE informatizado é uma tendência forte e atual para a profissão, uma vez que pode contribuir para aprimorar a qualidade e a segurança dos cuidados em saúde, estimular o pensamento reflexivo e ativo, facilitar o planejamento, a tomada de decisão, a comunicação, o controle gerencial e as mudanças na estrutura organizacional. Portanto, a necessidade de melhorar a avaliação do cliente no processo de cuidar em saúde se tornou ponto crucial no PE, para a garantia dos resultados na prática ${ }^{(1,5,7-8)}$.

Apesar dos esforços para sua informatização ou não, o PE ainda não tem sido totalmente incorporado na prática e a informática, por sua vez, não tem sido percebida como uma possibilidade real, integrada à complexidade da prática diária e muitos a consideram como mais uma tecnologia que se interpõe entre os profissionais e os clientes. Entretanto, estudos têm revelado que esta tecnologia da informação e comunicação revigora o pensamento crítico, o desenvolvimento do raciocínio clínico e investigativo e o fomento da busca contínua de informações que visam obter evidências científicas ${ }^{(1-2,6,9)}$.

Para que o PE seja desenvolvido e integrado a um sistema informatizado, o enfermeiro precisa estar fundamentado em uma base conceitual sólida ${ }^{(3)}$. A 
base conceitual da Enfermagem proposta neste estudo engloba a utilização de uma terminologia própria, considerando que a principal finalidade de uma terminologia estruturada e acordada entre os enfermeiros é demonstrar o valor da Enfermagem e sua contribuição na atenção à saúde. A utilização, portanto, de uma terminologia unificada e informatizada permite aos enfermeiros codificar, armazenar e recuperar a informação, em um formato que seja útil e aplicável na prática em terapia intensiva ${ }^{(7,10-11)}$.

Em razão disto, neste estudo, optou-se pela Classificação Internacional da Enfermagem (CIPE $\left.{ }^{\circledR}\right)$, versão 1.0, elaborada pelo Conselho Internacional dos Enfermeiros - ICN (International Council of Nursing) ${ }^{(11)}$, para a aprendizagem de enfermeiros com relação à implementação do PE informatizado na UTI. Considera-se que esta Classificação se constitui, a partir de outras terminologias existentes, em uma uniformização das terminologias científicas utilizadas pela Enfermagem e reúne, em uma única estrutura, os diagnósticos, as intervenções e os resultados que podem ser informatizados ${ }^{(11-12)}$.

Na sua essência, os componentes da CIPE ${ }^{\circledR}$ são os elementos da prática de Enfermagem, ou seja, estão relacionados ao fazer dos enfermeiros, em face de determinadas necessidades humanas, para produzir determinados resultados. Trata-se de uma linguagem unificada que expressa os elementos da prática de Enfermagem, permitindo comparações entre contextos clínicos, populações de clientes, áreas geográficas ou tempo. Também permite a identificação da Enfermagem em equipes multidisciplinares, a diferenciação da prática por níveis de preparação e experiência em Enfermagem, e ainda, possibilita o avanço nas correlações entre as atividades de Enfermagem e os resultados em saúde ${ }^{(11)}$

Diante deste cenário, para auxiliar os enfermeiros na aprendizagem da implementação do PE informatizado, alguns desafios se apresentam e precisam ser mais bem avaliados. Entre eles destacam-se: a pouca utilização da CIPE pelos enfermeiros brasileiros; o déficit no conhecimento desses profissionais para diagnosticar e realizar o cuidado baseado no diagnóstico levantado; o excesso de termos técnicos em língua inglesa utilizados pela informática e pelos sistemas de informação; a dificuldade em manusear os equipamentos de informática; bem como a falta de hábito dos enfermeiros em avaliar continuamente o resultado de sua prática diária(5,8,12-13)

Acredita-se na necessidade de os enfermeiros avaliarem melhor como a informática - e aqui, especialmente, os sistemas de informação, como o PE informatizado - é utilizada no cotidiano assistencial, questionando a si próprios sobre suas condutas diante dos clientes que demandam cuidados. Assim, entende-se que esses profissionais poderão adotar uma postura reflexiva de modo que possam fazer escolhas sobre qual o melhor uso de qualquer tecnologia introduzida no cuidado da Enfermagem em Terapia Intensiva.

Diante desta problemática, o presente estudo teve como objetivo avaliar a aplicação do Processo de Enfermagem informatizado, a partir da $\mathrm{CI} P E{ }^{\circledR}$ versão 1.0, com os enfermeiros da UTI de um Hospital Universitário do sul do Brasil.

\section{METODOLOGI A}

Foi desenvolvida uma pesquisa do tipo Convergente-Assistencial, que tem como fundamento básico a existência de articulação entre a prática assistencial ou educativa, no ambiente de trabalho, e o ofício investigativo, cujos resultados devem trazer contribuições que ajudem a melhorar esta mesma prática $^{(14)}$.

Para alcançar o objetivo proposto foi realizada uma prática educativa na UTI do Hospital Universitário da Universidade Federal de Santa Catarina, caracterizado como um hospital geral de grande porte da região metropolitana de Florianópolis - Santa Catarina, Brasil. Atualmente, a UTI possui 7 leitos para internação de pacientes clínicos e cirúrgicos e apresenta em seu quadro funcional oito enfermeiros, onze médicos, dezesseis técnicos de enfermagem, nove auxiliares de enfermagem e dois fisioterapeutas.

Participaram da prática educativa cinco enfermeiros que trabalham na UTI e que se dispuseram a tomar parte da pesquisa convergenteassistencial. O critério adotado para esta participação relacionava-se apenas à aceitação voluntária dos mesmos em participar do processo educativo, mediante conhecimento e assinatura do Termo de Consentimento Livre e Esclarecido. Três enfermeiros participaram dos encontros em seus respectivos turnos de trabalho e dois enfermeiros se disponibilizaram a participar em outros turnos. Não foi possível realizar os encontros coletivamente com todos os enfermeiros da UTI, devido ao excesso de demandas na unidade.

O estudo desenvolveu-se nos meses de outubro e novembro de 2007, constituindo-se de três encontros previamente agendados com os enfermeiros e de momentos de acompanhamento individual, de acordo com a disponibilidade dos participantes. Esses encontros foram desenvolvidos a partir da Metodologia Problematizadora de Paulo Freire ${ }^{(15)}$, sempre tendo como provocação reflexiva uma situação-problema exemplificada da realidade, que promovesse a sensibilização de todos os participantes para a temática, bem como possibilitasse o entendimento da proposta e a 
motivação para sua incorporação na prática. Cada encontro foi avaliado com os enfermeiros no sentido de compreender suas dificuldades no desenvolvimento do estudo e permitir o feedback quanto aos principais aspectos envolvidos na aplicação do Processo de Enfermagem informatizado, a partir da $\mathrm{CIPE}{ }^{\circledR}$ versão 1.0. Ao final da prática educativa, realizou-se então uma síntese da avaliação do PE informatizado, trazendo à tona as novas possibilidades e encaminhamentos, as dificuldades e facilidades que se apresentaram, assim como os pontos positivos e negativos relacionados ao assunto, mediante questionário previamente elaborado.

Para a operacionalização dos pressupostos da Metodologia Problematizadora de Paulo Freire, os encontros foram estruturados através do método denominado "Arco da Problematização", elaborado por Charles Maguerez ${ }^{(16)}$, envolvendo as etapas de observação da realidade e identificação do problema, determinação dos pontos chaves, teorização, hipóteses de soluções e aplicação à realidade, conforme relatado a seguir, de acordo com cada encontro.

O primeiro encontro envolveu as etapas de "Observação da Realidade e Identificação do Problema"; "Determinação dos Pontos-Chave" e "Teorização" que é a etapa de investigação propriamente dita. Neste momento foi realizada a apresentação dos participantes envolvidos, dos objetivos, dos conteúdos e da metodologia proposta para a prática educativa, com o intuito de sensibilizar e motivar a participação efetiva dos enfermeiros. Nesta oportunidade também foi feito amplo levantamento e reflexão sobre o PE desenvolvido na UTI e a possibilidade de inserção da informática em Enfermagem neste contexto, reforçando com o grupo de participantes a observação da realidade vivenciada na UTI. Foi distribuído material de referência para leitura e fundamentação, secundado por discussões e reflexões do(s) problema(s) ou tema(s) levantado(s), destacando-se, por meio de questões norteadoras, os aspectos principais da $\mathrm{CIPE} \circledast$ e o PE (momento este que reforçou a etapa da determinação dos pontos-chave do arco da problematização).

Ainda, à luz das crenças, valores, conhecimentos, experiências, reflexões e discussões desses profissionais, buscou-se o entendimento e a compreensão do PE informatizado e da $\mathrm{CIPE} \circledast$, com aprofundamento teórico (teorização) associado à realidade da UTI. Finalmente, foi entregue aos enfermeiros um caso clínico simulando a realidade do cuidado em UTI, para que os mesmos pudessem compor os diagnósticos, as intervenções e os resultados do cuidado de enfermagem, de acordo com a CIPE ${ }^{\circledR}$.
É importante ressaltar que, embora as etapas do arco da problematização de Maguerez tenham sido descritas aqui, separadamente, apenas por razões redacionais, na prática com os enfermeiros, elas aconteceram em convergência, ou seja, numa articulação metodológica em que a prática gerava problemas, a partir da observação e vivência com a realidade, focalizando a temática central do estudo. Este exercício, por sua vez, era alimentado e aprofundado pela teoria, na busca de soluções ${ }^{(16)}$.

O segundo encontro, que destacou a etapa de "Hipóteses ou Soluções" do arco, resgatou as dúvidas levantadas na discussão teórica realizada no encontro anterior. Discutiu-se também sobre a composição dos diagnósticos e as intervenções de enfermagem segundo a $\mathrm{CIPE}{ }^{\circledR}$, a partir do caso clínico simulado distribuído previamente. Foram ainda apresentados os critérios para avaliação do sistema informatizado (ergonomia, conteúdo e usabilidade), conforme instrumento fundamentado na ISO (International Standard Organization) 9241-11, entidade que estabelece os Guidelines de Usabilidade dos sistemas de informação ${ }^{(17)}$, e realizado o cadastramento dos enfermeiros na plataforma computadorizada, que atualmente está disponível em: http://www.nfrinfor.ufsc.br.

A partir deste cadastramento houve oportunidade para esclarecer aos participantes do estudo as dúvidas sobre o sistema (estrutura dos dados, registro, telas), a navegação pela plataforma computadorizada e o aprofundamento na estruturação dos dados. O grupo foi ainda orientado a simular outros casos clínicos na plataforma, tanto de seu próprio local de trabalho, quanto de seu domicílio, uma vez que o sistema permitia acesso via Web mediante login e senha de acesso.

Finalmente, no terceiro encontro, buscou-se a etapa de "Aplicação à Realidade", com a operacionalização, na prática da UTI, do PE informatizado, utilizando-se então uma situação real. Neste momento, também foram realizados os esclarecimentos das dúvidas em relação ao sistema e à estrutura dos dados. O questionário de avaliação foi preenchido pelos participantes e contou com a orientação de uma das autoras do estudo. As orientações foram no sentido de identificar as principais contribuições da proposta, o que poderia ser melhorado no PE informatizado, quais as expectativas em relação à possibilidade de disponibilizar também em uma plataforma computadorizada móvel o PE de enfermagem, bem como quais os pontos positivos e negativos apresentados durante a realidade desta prática educativa.

Salienta-se que foram respeitados os preceitos éticos determinados pela resolução №196/96 do Conselho Nacional de Saúde, mediante o 
cumprimento das exigências do Termo de Consentimento Livre e Esclarecido, por meio do direito de informação da pessoa e do respeito à liberdade dos participantes para que pudessem, a qualquer momento, desistir do estudo. Tal Consentimento foi obtido no primeiro encontro. Durante o desenvolvimento dos encontros foi solicitado ao grupo autorização para registro escrito e gravação das informações e observações levantadas. Este estudo foi submetido ao Comitê de Ética da Universidade Federal de Santa Catarina e aprovado, sem ressalvas, pelos membros da entidade em 03 de outubro de 2007 de acordo com o protocolo no. 0264/07.

As autoras do estudo comprometeram-se a não expor os participantes acerca das informações e observações levantadas durante os encontros, estabelecendo uma relação de confiança durante o desenvolvimento do mesmo. Para tanto, os enfermeiros participantes deste estudo foram identificados como Enfo 1, Enfo2... e assim sucessivamente.

De acordo com o referencial teórico adotado ${ }^{(15-}$ 16) e com a sistematização dos encontros, a avaliação da prática educativa foi processual, durante o seu desenvolvimento, e também durante um momento específico final, com o auxílio de um questionário previamente elaborado(17). Os dados foram analisados de forma quantitativa, por meio de estatística descritiva, e qualitativamente, de acordo com o referencial teórico adotado. Esta avaliação também permeou o desenvolvimento de todo o estudo, num ir e vir dinâmico e constante, buscando responder ao objetivo proposto.

\section{RESULTADOS E DISCUSSÃO}

No primeiro encontro desta prática educativa com os enfermeiros da UTI, três questionamentos centrais conduziram a atividade. Tais perguntas buscaram relacionar o trabalho proposto com a realidade vivenciada na referida unidade, bem como estabelecer os pontos-chave para a discussão e a teorização.

O primeiro questionamento sobre a realidade foi: "quais as etapas do Processo de Enfermagem que você realiza na UTI?". As seguintes respostas foram obtidas de acordo com o enfo 2 e enfo 4: coleta de dados, histórico de enfermagem, evolução de enfermagem e prescrição de enfermagem. A gente ainda não utiliza o diagnóstico de enfermagem e, bom, o plano de enfermagem acaba entrando dentro da evolução de enfermagem. Sempre que faz a evolução é o método WEED, em forma do SOAP; então o plano de cuidados entra dentro da nossa evolução. E a prescrição de enfermagem é separada, diferenciada. Não usamos o diagnóstico de enfermagem ainda (ENFo 2).; e [...] uso três etapas.
Só o histórico de enfermagem para levantamento de dados, a intervenção de enfermagem que seria a prescrição de enfermagem, e a evolução. A parte de diagnóstico não tem um método específico, um lócus específico dentro do nosso processo para desenvolvimento de diagnóstico ou avaliação dos dados levantados. Na realidade ele pula direto para a parte de intervenção (ENFo 4).

Importante ressaltar que a base conceitual adotada na UTI do estudo para o desenvolvimento do Processo de Enfermagem é a teoria das Necessidades Humanas Básicas (NHB) de Wanda Horta. Este referencial contempla, na lógica de sua estrutura, 05 etapas do PE e, dentre elas, a do diagnóstico de enfermagem. Como exemplo, pode-se citar a 'necessidade de oxigenação alterada'. Trata-se de um diagnóstico específico de Wanda Horta contemplado no seu referencial e não em outra terminologia, assim como ocorre com a $\mathrm{CIPE}{ }^{\circledR}$ ou a nomenclatura diagnóstica da NANDA (North American Nursing Diagnosis Association), que integram termos específicos. Isso remete à reflexão de que não há necessidade de se suprimir as etapas do Processo de Enfermagem já desenvolvidas nesta unidade do estudo, especialmente porque elas fazem parte do próprio referencial adotado na Instituição, mas, sobretudo, de adequá-la a uma nova terminologia que pode ser informatizada. O diagnóstico, portanto, é peça fundamental do Processo de Enfermagem porque ele é a reunião de dados para o julgamento clínico de Enfermagem que direciona as ações de cuidado ${ }^{(18)}$.

As fases de coleta de dados, diagnóstico, planejamento, implementação e avaliação se relacionam e dependem uma da outra, sendo inseparáveis dentro de um contexto prático. Entendese que, especificamente na UTI, onde a situação clínica do cliente é instável, o Processo de Enfermagem (PE), desenvolvido em todas as suas etapas, facilitará o domínio clínico da tomada de decisão para um cuidado mais qualificado, seguro e centrado nas necessidades dos clientes ${ }^{(9,19)}$.

Neste sentido, compreende-se que o diagnóstico de enfermagem, enquanto enunciado, é a agregação dos dados que compõem a avaliação clínica realizada pelos enfermeiros, ou seja, considera-se que quanto maior o número de necessidades afetadas do cliente, maior será a necessidade de se planejar a assistência baseada nos diagnósticos levantados, uma vez que a sistematização das intervenções visa a organização, a eficiência e a validade do cuidado prestado.

O segundo questionamento realizado aos enfermeiros sobre "quais as principais dificuldades encontradas na aplicação do Processo de Enfermagem na UTI?" possibilitou identificar a sobrecarga e o acúmulo de atividades, a falta de tempo e o número insuficiente de enfermeiros na 
unidade: o que eu tenho dificuldade em relação à aplicação do processo mesmo, evolução, a própria coleta de dados, o histórico de enfermagem, primeiro é a questão do tempo. Muitas vezes você está como enfermeiro sozinho e tem uma série de intercorrências, funcionários novos, toda a questão de supervisão, muitas vezes a gente tem alunos, [...] eles demandam um pouco mais de cuidados, de supervisão um pouco mais direta. Então a gente acaba demandando um pouco mais de tempo para outras coisas em detrimento ao processo de enfermagem (ENFO2).; e [...] Entre as principais dificuldades estão a falta de tempo, a sobrecarga de trabalho [...], acúmulo de atividades (ENFo3).

Estudos evidenciam que o registro eletrônico afeta a eficiência do tempo na prática, especialmente o tempo para a documentação(2). Por outro lado, outro estudo afirma que o registro eletrônico reduz o tempo de documentação dos enfermeiros na prática $^{(5)}$. Neste sentido, o sucesso ou o fracasso de um sistema de informação clínica, na prática, relaciona-se com a capacidade do sistema de integrar-se ao ambiente de trabalho, às práticas, e às necessidades de informação dos usuários ${ }^{(20)}$.

Constata-se ainda que a formação do enfermeiro requer e exige capacitações e atualizações permanentes, de modo a fomentar/incrementar uma prática de cuidado baseada em evidências, a fim de que haja melhor embasamento para a tomada de decisão, a discussão clínica entre os pares, e a experiência do próprio profissional. Pode-se dizer, portanto, que há uma urgente necessidade de que sejam revistos os aspectos da formação profissional, não apenas na área de terapia intensiva, mas de modo geral.

Os participantes interpretam que a metodologia da assistência atualmente desenvolvida pelo PE na UTI do estudo parece estar deslocada das necessidades do cliente, ou seja, os registros efetuados, muitas vezes estão dissociados da sua situação clínica, estão sendo executados apenas como um dever, e não como um reflexo das necessidades do cliente e dos resultados da prática do enfermeiro. Esta percepção pode ser confirmada na seguinte fala: acho que a prescrição de enfermagem hoje está deixando um pouco a desejar. Dos dois lados, na parte de execução e na parte de elaboração. [...] como o método de assistência hoje está um pouco desatualizado, com o tempo os enfermeiros acabam não implementando situações novas ou implementam e não trazendo para o grupo. Então tem um mescla muito grande de velhas e novas vertentes aqui dentro (ENFo 4).

A desatualização do método de assistência mencionada na fala do ENFo4 pode estar relacionada à ausência ou falta de retorno à aplicação de uma metodologia que contemple o cuidado voltado para as condições e necessidades do cliente de terapia intensiva ${ }^{(12-13)}$.

O terceiro questionamento feito foi: "qual sua opinião sobre a informática na Enfermagem?". Nesta realidade observada foi possível constatar que os enfermeiros consideram a informática como uma aliada da profissão, porém ressaltam que sua utilização deve ser de forma integrada e que atenda às necessidades da demanda de trabalho, evidenciada nas seguintes falas: eu acho que, na verdade, a informática é um recurso que não tem como fugir, principalmente na área enfermagem; otimizando recurso humano, recurso material, a questão do tempo também, a economia de tempo. A questão de integração daquilo que o enfermeiro pode pensar e resgatar daquilo que a informática pode oferecer. [...] Não acredito em ponto negativo. Acredito que é mais um recurso que tem a oferecer bons resultados que pode ajudar e contribuir bastante (ENFo 4).; e aí depende. Depende de como que esta base onde você vai alimentar. O que nós temos hoje, se a gente for fazer um histórico de enfermagem pelo sistema que nós temos hoje a informática atrapalha porque ela não é completa. [...] é para paciente clínico. É para o hospital todo, não é só para a UTI. [...] A gente fazia (a prescrição) manuscrita anteriormente e depois nós passamos a fazer dentro de um documento, circulando ou sublinhando aquilo que preciso para se observar, mas acabava tendo muito chavão e coisas muito perdidas dentro de outras coisas que não eram observadas, que não deveriam ser observadas para aquele paciente (ENFo 2).

Percebe-se que a informática acaba por dificultar a implantação do Processo de Enfermagem informatizado, especialmente pelo modo como vem sendo utilizada atualmente na instituição do estudo, uma vez que não há uma metodologia de enfermagem específica para a UTI, e sim, para toda a unidade hospitalar. O "copiar e colar" (destaque das autoras) de um arquivo para outro, que ocorre frequentemente na prescrição de enfermagem, não está relacionado com o cliente específico da UTI; não há espaço para o histórico de enfermagem, evolução, entre outros.

Dito de outro modo, no referido hospital, a estruturação dos dados na metodologia contempla o histórico de enfermagem e as prescrições gerais para a maioria das unidades, com exceção da unidade de pediatria. A UTI, objetivando aperfeiçoar a prescrição de enfermagem, adaptou esta estrutura selecionando as intervenções específicas para a unidade, copiandoas em arquivo de editor de texto Word ${ }^{\circledR}$ e, posteriormente, retornando-as e inserindo-as no sistema do hospital, o que não caracteriza o PE desenvolvido na unidade como informatizado. 
A partir das três questões norteadoras sobre a "observação da realidade" foi possível determinar, juntamente com os enfermeiros, os "pontos-chave" para discussão e "teorização" desta prática educativa. Os pontos-chave levantados foram: sistemas de informação e informática na enfermagem; processo de enfermagem informatizado e; Classificação Internacional para a Prática de Enfermagem (CIPE ${ }^{\circledR}$ versão 1.0). Ao último ponto-chave foi dada maior ênfase, por se tratar do elemento central que permeou toda a prática educativa, e por ser um sistema de classificação da prática de Enfermagem pouco utilizado no Brasil. Toda a teorização foi apresentada no aplicativo Power Point ${ }^{\circledR}$, procedida de momentos de discussão e reflexão sobre o pontochave abordado.

No 2 o encontro, os enfermeiros foram orientados para compor os diagnósticos, os resultados e as intervenções de enfermagem, segundo a $\mathrm{CIPE} \circledast$ versão 1.0. Com a intenção de demonstrar como esta classificação se aplicava no cotidiano da prática de enfermagem, o caso clínico foi analisado a partir do referido sistema de classificação.

Um questionário para avaliação do Processo de Enfermagem informatizado segundo a $\mathrm{CIPE}{ }^{\circledR}$ versão 1.0 foi distribuído no 3 o encontro, contemplando sua avaliação segundo os aspectos de ergonomia e usabilidade ${ }^{(17)}$. Os dados obtidos foram tabulados e são apresentados nas tabelas seguintes. Destaca-se, neste momento, que os cinco enfermeiros participaram da prática educativa, no entanto, um deles não realizou a avaliação do PE informatizado.

Na avaliação ergonômica abordada na Tabela 1, utilizaram-se três critérios de avaliação: organização (03 itens), interface (04 itens) e técnico (03 itens). Os itens foram distribuídos em uma escala de valores com as seguintes categorias de respostas: (5) Excelente, (4) Muito Bom, (3) Bom, (2) Regular, (1) Ruim. Considerou-se na avaliação que os valores da média entre: 1 a 1,5 (receberiam a classificação Ruim); de 1,51 a 2,5 (Regular); de 2,51 a 3,5 (Bom); de 3,51 a 4,5 (Muito Bom) e de 4,51 a 5 (Excelente).

Tabela 1: Avaliação do processo de enfermagem informatizado pelos enfermeiros participantes, segundo o aspecto ergonômico - Critérios de Organização, Interface e Técnico. Florianópolis-SC, 2008.

\begin{tabular}{|c|c|c|c|c|c|c|}
\hline \multirow{2}{*}{ I tens Avaliados } & \multicolumn{4}{|c|}{ Participantes } & \multirow{2}{*}{$\begin{array}{l}\text { Média entre os } \\
\text { sujeitos }\end{array}$} & \multirow{2}{*}{$\begin{array}{l}\text { Média do } \\
\text { item }\end{array}$} \\
\hline & 1 & 2 & 3 & 4 & & \\
\hline \multicolumn{7}{|l|}{ Organização } \\
\hline $\begin{array}{l}\text { A organização e o modo de acesso aos arquivos do sistema } \\
\text { favorecem a execução do Processo de Enfermagem }\end{array}$ & 4 & 4 & 3 & 3 & 3,5 & \multirow{3}{*}{3,7} \\
\hline O sistema apresenta facilidade de operacionalização & 4 & 4 & 3 & 3 & 3,5 & \\
\hline $\begin{array}{l}\text { O sistema alcança seu objetivo - desenvolver o Processo de } \\
\text { Enfermagem em Terapia Intensiva utilizando a CIPE }\end{array}$ & 5 & 4 & 3 & 4 & 4,0 & \\
\hline \multicolumn{7}{|l|}{ Interface } \\
\hline Aparência das telas é adequada & 4 & 4 & 3 & 3 & 3,5 & \multirow{4}{*}{3,3} \\
\hline $\begin{array}{l}\text { Os dados, os diagnósticos e as intervenções levantadas no } \\
\text { sistema são visualmente acessíveis e de fácil entendimento } \\
\text { A quantidade de informação é suficiente para a assistência de }\end{array}$ & 4 & 2 & 3 & 2 & 2,7 & \\
\hline $\begin{array}{l}\text { enfermagem (coleta de dados, diagnósticos e intervenções) } \\
\text { podendo ser utilizado em diferentes tipos de UTI }\end{array}$ & 4 & 5 & 3 & 3 & 3,7 & \\
\hline \multicolumn{6}{|l|}{ Técnico } & \\
\hline $\begin{array}{l}\text { Estrutura dos dados - como os dados no sistema foram } \\
\text { organizados permitindo um raciocínio compatível com a } \\
\text { prática }\end{array}$ & 3 & 4 & 3 & 4 & 3,5 & \multirow{4}{*}{3,6} \\
\hline O sistema oferece segurança e privacidade das informações & & & & & & \\
\hline $\begin{array}{l}\text { não permitindo, por exemplo, mudar os dados após serem } \\
\text { salvos, exigindo login e senha de acesso de entrada. }\end{array}$ & 3 & 3 & 3 & 4 & 3,2 & \\
\hline O sistema funciona adequadamente pela internet. & 3 & 4 & 4 & 5 & 4,0 & \\
\hline
\end{tabular}

Observa-se que o critério de Organização do sistema foi considerado "Muito Bom". Tal aspecto permite assinalar que o sistema tem facilidade de manuseio, acesso e, ao mesmo tempo, atinge o objetivo central proposto de desenvolver $\mathrm{O} P \mathrm{PE}$ informatizado utilizando a $\mathrm{CIPE}{ }^{\circledR}$. Isto pode estar relacionado tanto à facilidade quanto ao hábito dos participantes em manusear os recursos da Internet, uma vez que a UTI do estudo disponibiliza este acesso aos profissionais desta unidade. Além disso, o sistema não requer instalação de programas complexos, tampouco estudos de manuais quanto à lógica de estrutura dos dados no ambiente informatizado, o que na opinião das autoras, aproxima-se da realidade prática. 
No que diz respeito ao critério Interface do sistema, o mesmo foi caracterizado com escore "Bom", demonstrando que o sistema é de fácil visualização e apresenta informação em quantidade suficiente para o desenvolvimento do PE em diferentes tipos de UTI. Contudo, o item "os dados, os diagnósticos e as intervenções levantados no sistema são visualmente acessíveis e de fácil entendimento" recebeu escore 2 por dois participantes, diminuindo sua média para 2,7. Um sistema de informação para clientes em situações de saúde críticas, complexas e de risco em ambiente de terapia intensiva exige um volume grande de informações a serem avaliadas pelo profissional. Há, portanto, dificuldades de incorporar e entender a complexa estrutura e lógica destas informações por grande parte dos enfermeiros.

O sistema ora desenvolvido para a UTI incluiu o máximo possível de informações para ser adaptado em qualquer unidade intensiva, de acordo com as características dos clientes. Ao mesmo tempo, oferece aos enfermeiros um leque de possibilidades, logicamente estruturadas, em uma terminologia de referência para apoio ao julgamento e ao cuidado de Enfermagem, de acordo com a natureza que as situações clínicas exigem ${ }^{(20)}$.

O critério Técnico da avaliação ergonômica também foi considerado "Muito Bom" pelos enfermeiros, demonstrando que a estruturação dos dados, a segurança e privacidade das informações e seu funcionamento pela internet são adequados e satisfatórios.

Vale ressaltar que, desde os primeiros estudos, iniciados ainda em 1999, sobre o sistema informatizado implementado neste estudo, o critério técnico de segurança e privacidade das informações foi criteriosamente adotado pela equipe de desenvolvimento do sistema, visando a garantia da segurança e da ética da informação, que perpassam a assistência de enfermagem nos mais variados cenários de assistência à saúde ${ }^{(3,18)}$. Para assegurar esta propriedade do sistema, cada participante possuía um login e uma senha individual e intransferível, que lhe garantiam autonomia e segurança no registro dos dados e das informações, sem interferir na dos demais participantes do estudo.

Na Tabela 2 são apresentados os resultados da avaliação que os enfermeiros fizeram relativos aos aspectos Conteúdo e Usabilidade do processo de enfermagem informatizado. Observa-se que, para o critério Conteúdo, os participantes atribuíram uma média geral que o caracteriza como "Muito Bom. O conteúdo é entendido neste estudo como a capacidade de o sistema avaliar adequadamente, a partir dos dados estruturados, se as informações estão objetivas, e atualizadas de acordo com as situações clínicas apresentadas pelos clientes, bem como, se o próprio conteúdo contempla a área da UTI.

Tabela 2: Avaliação do processo de enfermagem informatizado pelos enfermeiros participantes, segundo os aspectos do conteúdo e usabilidade. Florianópolis-SC, 2008.

\begin{tabular}{|c|c|c|c|c|c|c|}
\hline \multirow{2}{*}{ I tens Avaliados } & \multicolumn{4}{|c|}{ Participantes } & \multirow{2}{*}{$\begin{array}{l}\text { Média entre os } \\
\text { sujeitos }\end{array}$} & \multirow{2}{*}{$\begin{array}{l}\text { Média do } \\
\text { item }\end{array}$} \\
\hline & $\mathbf{1}$ & 2 & 3 & 4 & & \\
\hline \multicolumn{7}{|l|}{ Conteúdo } \\
\hline As informações são claras, objetivas e atualizadas & 3 & 5 & 3 & 4 & 3,7 & \multirow[b]{2}{*}{3,9} \\
\hline $\begin{array}{l}\text { O conteúdo está inter-relacionado e consistente com a } \\
\text { área de UTI. }\end{array}$ & 3 & 5 & 3 & 5 & 4,0 & \\
\hline \multicolumn{7}{|l|}{ Usabilidade } \\
\hline $\begin{array}{l}\text { O programa roda facilmente na plataforma, sem } \\
\text { interferências }\end{array}$ & 3 & 4 & 4 & 5 & 4,0 & \multirow{8}{*}{3,9} \\
\hline As telas do sistema são claras, fáceis de ler e interpretar & 3 & 4 & 3 & 4 & 3,5 & \\
\hline $\begin{array}{l}\text { As instruções de apoio ao usuário são claras, organizadas } \\
\text { e bem implementadas }\end{array}$ & 3 & 5 & 3 & 4 & 3,7 & \\
\hline $\begin{array}{l}\text { O usuário é capaz de acessar o sistema/programa } \\
\text { facilmente }\end{array}$ & 3 & 5 & 5 & 5 & 4,5 & \\
\hline O menu é viável e fácil de usar & 3 & 4 & 3 & 5 & 3,7 & \\
\hline $\begin{array}{l}\text { O menu atende as funções definidas para os objetivos do } \\
\text { sistema }\end{array}$ & 3 & 5 & 3 & 5 & 4,0 & \\
\hline $\begin{array}{l}\text { É eficiente para o intercâmbio de informação entre o } \\
\text { usuário e o programa }\end{array}$ & 3 & 4 & 3 & 4 & 3,5 & \\
\hline O sistema está integrado a um banco de dados & 3 & 5 & 4 & 4 & 4,0 & \\
\hline
\end{tabular}

Entende-se que o conteúdo presente na plataforma computadorizada deve abranger todos os dados necessários para a avaliação adequada dos clientes em tratamento intensivo, bem como oportunizar ao enfermeiro a tomada de decisão mais segura em relação ao cuidado por interagir com um 
ambiente tecnológico de informação estruturada. Por conta disso, no desenvolvimento deste sistema de informação foram construídos aproximadamente 825 diagnósticos de enfermagem e 1.600 intervenções de enfermagem estruturados a partir da CIPE® versão 1.0, com o objetivo de contemplar todos os dados e as informações necessárias para a implementação do PE informatizado em terapia intensiva.

A avaliação da Usabilidade do sistema, que mede a eficácia, a eficiência e a satisfação com que o enfermeiro pode realizar um conjunto específico de tarefas em um ambiente particular, resultou no conceito "Muito Bom", explicitando que o menu do sistema é facilmente acessado, viável e atende às funções e objetivos do PE, bem como estabelece de forma eficiente o intercâmbio de informações entre os usuários e o programa.

Tais resultados podem ser observados através dos aspectos positivos e negativos do sistema, ressaltados pelos enfermeiros. Entre os aspectos positivos destacam-se: fácil navegação; direcionado à UTI, contempla os passos propostos; fácil visualização dos problemas finais (ENFo4) e utilização da informática na enfermagem (tendência futura); uniformização do processo de enfermagem; atende a exigência legal quanto a anotações, registros legíveis (ENFo1). Os aspectos negativos apontados foram: relativamente extenso (ENFo4); e muitos diagnósticos afins; ao utilizar o sistema contendo todos os itens - tempo elevado $X$ difícil para aplicação prática (ENFo 1).

No que diz respeito aos aspectos negativos do sistema ressalta-se que o volume elevado de diagnósticos foi propositalmente levantado, de modo a permitir uma aproximação e uma identificação da terminologia CIPE com o maior número possível de enfermeiros, para facilitar sua incorporação na prática.

Na Tabela 3 é apresentada a avaliação de alguns itens do critério Usabilidade, que foram analisados separadamente, porque não foram avaliados pelo Enfermeiro 1. Evidencia-se que o sistema possui salvamento automático, segurança de dados e recuperação dos dados nos vários módulos, tendo sido considerado “Muito Bom" pelos enfermeiros.

Tabela 3: Avaliação do processo de enfermagem informatizado pelos enfermeiros participantes, segundo itens do critério Usabilidade que não foram avaliados por todos os participantes. Florianópolis-SC, 2008.

\begin{tabular}{|c|c|c|c|c|c|c|}
\hline \multirow{2}{*}{ Itens de Avaliação } & \multicolumn{4}{|c|}{ Participantes } & \multirow{2}{*}{$\begin{array}{l}\text { Média entre } \\
\text { os sujeitos }\end{array}$} & \multirow{2}{*}{$\begin{array}{l}\text { Média do } \\
\text { item }\end{array}$} \\
\hline & 1 & 2 & 3 & 4 & & \\
\hline \multicolumn{7}{|l|}{ Usabilidade } \\
\hline O sistema possui salvamento automático & - & 3 & 5 & 4 & 4,0 & \multirow{4}{*}{3,6} \\
\hline O sistema possui segurança de dados & - & 4 & 5 & 3 & 4,0 & \\
\hline O sistema não aceita dados inexistentes & - & 3 & 3 & 4 & 3,3 & \\
\hline $\begin{array}{l}\text { O sistema permite a recuperação dos dados nos vários } \\
\text { módulos }\end{array}$ & - & 4 & 3 & 2 & 3,0 & \\
\hline
\end{tabular}

Ressalta-se que a utilização de sistemas de informação computadorizados na assistência de Enfermagem exige avaliação contínua quanto à sua eficácia e aplicabilidade. Tal avaliação relaciona-se aos critérios de Ergonomia, Conteúdo e Usabilidade e, portanto, o referencial da problematização adotado neste estudo, permitiu um re-visitar contínuo e dinâmico à evidência da prática de Enfermagem, ou seja, o referencial da problematização fundamenta o olhar crítico e contínuo dos critérios de qualidade exigidos para um sistema de informação, neste estudo, o PE informatizado. Estes parâmetros de avaliação devem sempre acompanhar o desenvolvimento dos sistemas informatizados, pois, além de estarem em conformidade com as exigências do padrão ISO $9241-11^{(17)}$, eles também mostram que o sistema pode ser usado na prática assistencial, associado com a realidade vivenciada pelos profissionais.

Todos os enfermeiros do estudo avaliaram que a CIPE ${ }^{\circledR} 1.0$ é aplicável ao PE informatizado em terapia intensiva, como pode ser constatado nos seguintes depoimentos: [...] facilidades para implementar o processo (ENFo 3); e utiliza raciocínio simples e prático (ENFo4). Todos os enfermeiros também consideraram que o PE informatizado em UTI pode ser um caminho para aproximá-los do cuidado direto porque exige avaliações clínicas contínuas e progressivas dos clientes, conforme relatado na fala: esta relação é dinâmica e eficiente no momento em que o processo é moldado às necessidades reais do cuidado (ENFo4).

Outra questão que emergiu durante 0 desenvolvimento do estudo foi o "tempo disponível" (destaque das autoras) para realização do PE informatizado, segundo a $\mathrm{CIPE}{ }^{\circledR}$ 1.0. A fala do Enfermeiro 2 expressa esta preocupação: o registro é importante, mas o tempo utilizado para ele deve ser o mínimo possível, visto que o enfermeiro precisa ter mais tempo para implementar as ações/supervisionar, etc. (ENFo2).

Buscando hipóteses de solução para o problema levantado, os enfermeiros enfatizaram que o tempo é um fator extremamente importante, uma vez que o 
volume de dados e de informações precisa ser o maior possível, a fim de contemplar as mais variadas realidades das UTIs. Torna-se necessário, contudo, que os enfermeiros se familiarizem com todos os dados do sistema, de modo a direcionar a avaliação clínica de enfermagem para as prioridades clínicas dos clientes. Portanto, em outra etapa do processo de avaliação, a estrutura dos dados contemplados no sistema poderá ser organizada, de acordo com a UTI do hospital em questão.
O Quadro 1 apresenta as principais dificuldades apontadas pelos enfermeiros ao manusear o sistema do PE informatizado. As dificuldades relacionadas ao levantamento dos dados e à interpretação dos resultados radiológicos e laboratoriais podem estar associadas ao déficit da formação do enfermeiro nesta área específica, tanto pela academia, que coloca pouca ênfase na avaliação desses resultados, quanto pela própria falta de hábito deste profissional de incorporar tais dados na avaliação clínica do cliente.

Quadro 1: Dificuldades identificadas pelos enfermeiros durante o manuseio do sistema informatizado. Florianópolis-SC, 2008.

\begin{tabular}{|c|c|}
\hline DI FI CULDADES & Freq. ( N) \\
\hline Tempo para fazer o Processo de Enfermagem completo & 3 \\
\hline Avaliar os resultados da assistência a partir da CIPE & 3 \\
\hline Interpretação dos exames laboratoriais e radiológicos & 2 \\
\hline Associar a CIPE ao Processo de Enfermagem informatizado & 2 \\
\hline Levantamento dos dados & 1 \\
\hline Estabelecimento dos diagnósticos & 1 \\
\hline Seleção das intervenções & 1 \\
\hline Entender a estruturação e a lógica dos dados e das informações no sistema & 1 \\
\hline
\end{tabular}

Embora possa parecer que esses dados sejam incoerentes com os dados de relativos à Ergonomia e Usabilidade levantados, bem como com a questão da CIPE ${ }$ ter sido considerada uma classificação aplicável ao PE informatizado, percebe-se a necessidade de tempo maior para manusear, aplicar e relacionar o sistema com a situação clínica do cliente. Acredita-se que o fato de a CIPE ${ }^{\circledR}$ ser uma terminologia nova no Brasil, iniciada somente em 1999, tenha contribuído para as principais dificuldades encontradas pelos enfermeiros.

Ao se retomar a questão da falta de tempo que emerge frequentemente nas falas e registros dos enfermeiros, como um dos critérios que dificultam o desenvolvimento desta tecnologia na prática, entende-se que o PE é a assistência de Enfermagem na sua forma objetiva, contemplando intervenções de cuidado e registros e, portanto, estas dimensões não devem estar dissociadas. Isto é o mesmo que reiterar que o cuidado deve estar refletido no registro da informação e vice-versa. Esta constatação enfatiza a necessidade de priorizar o PE na UTI como estratégia precípua para a melhoria da qualidade do cuidado e da própria visibilidade profissional. Por outro lado entende-se que, ao priorizar estes aspectos, haverá necessidade de reorganizar as demandas na UTI. Consequentemente, o fator "tempo" estará sendo conduzido pela prioridade da assistência e pela medida de resultados, e não apenas como volume e carga de trabalho e/ou acúmulo de atividades.

\section{CONSI DERAÇÕES FI NAIS}

O referencial teórico adotado neste estudo, operacionalizado pelo Arco da Problematização, permitiu aproximar o objeto da pesquisa à realidade prática e, com isso, gerar dados que acabaram por refletir os resultados alcançados. Embora o estudo tenha sido desenvolvido com um número reduzido de participantes, não permitindo, portanto, sua generalização, compreende-se que 0 mesmo possibilitou o envolvimento, a colaboração e a participação ativa dos enfermeiros para a melhoria de sua prática.

Esta dinâmica, fundamentada na problematização, permitiu ainda resgatar os saberes científicos dos participantes, estimulando a que os mesmos contribuíssem efetivamente para a aplicação do PE informatizado na UTI e, sobretudo, o desenvolvimento de uma prática dialógica, crítica e reflexiva.

Os resultados permitem ressaltar que o sistema informatizado proposto para a aplicação do PE possui critérios de ergonomia e usabilidade compatíveis com os padrões internacionais exigidos para o desenvolvimento de sistemas. Exige-se, contudo, que sejam desenvolvidos novos estudos, abrangendo um número maior de participantes para sua implementação na Enfermagem de terapia intensiva.

A $C I P E \otimes$, enquanto terminologia de referência para a Enfermagem, revelou, para os enfermeiros do estudo, algumas características que facilitam sua aplicação. Tais características relacionam-se à facilidade de entendimento, visto que sua linguagem é compreensível, adequada e próxima da realidade 
da prática intensivista. Além disso, incorpora, em uma única classificação, os diagnósticos, as intervenções e os resultados e, com isso, permite o desenvolvimento da prática de Enfermagem baseada na evidência em terapia intensiva.

Estudos têm revelado que os sistemas informatizados representam o maior investimento de recursos de capital e humano em todo o mundo e, consequentemente, afetam os profissionais de saúde nos mais variados cenários de cuidado. Sendo assim, os profissionais têm o potencial tanto para melhorar quanto para criar obstáculos ao desempenho do trabalho, à comunicação e à documentação. Os enfermeiros são componentes fundamentais do cuidado em saúde e, portanto, torna-se necessário o envolvimento e a atuação permanente dos pesquisadores e profissionais no desenvolvimento e aplicação de sistemas de informação, pois estes terão resultado direto sobre o desempenho dos enfermeiros na prática, sobretudo, nos resultados aplicados à qualidade da assistência aos clientes. Há necessidade de ampliar estudos neste sentido.

Por fim, entende-se a importância de estudos desta natureza para o avanço da enfermagem brasileira, uma vez que tal projeto ultrapassa o exercício apenas intelectual, revertendo-se em execuções e encaminhamentos práticos de forma colaborativa e também autônoma. Ao evidenciar o compromisso e o comprometimento dos participantes com sua prática, componentes políticos e sociais são explicitados, pois a partir do contexto observam seus problemas e dificuldades, e para o contexto retornam, propondo respostas e soluções, visando transformar a realidade cotidiana, em direção à melhoria da qualidade científica, tecnológica e humana, no cenário da terapia intensiva.

\section{REFERÊNCI AS}

1. Zuzelo PR, Gettis C, Hansell AW, Thomas L. Describing the influence of technologies on registered nurses' work. Clin Nurse Spec. 2008; 22(3): 132-40.

2. Moody LE, Slocumb E, Berg B, Jackson D. Electronic health records documentation in nursing: nurses' perceptions, attitudes, and preferences. Comput Inform Nurs. $2004 ; 22(6)$ : 337- 44.

3. Antunes CR. Processo de enfermagem informatizado ao paciente politraumatizado de terapia intensiva via web [dissertation]. Florianópolis: Programa de PósGraduação em Enfermagem/UFSC; 2006. 151 p.

4. Kossman SP, Scheidenhelm S. Nurses' perceptions of the impact of electronic health records on work and patient outcomes. Comput Inform Nurs. 2008;26(2): 6977.

5. Hakes B, Whittington J. Assessing the impact of an electronic medical record on nurse documentation time. Comput Inform Nurs. 2008;26(4): 234-41.
6. Kossman SP. Perceptions of impact of electronic health records on nurses' work. Stud Health Technol Inform. 2006; 122: 337-41.

7. Organización Panamericana de la Salud. Desarrollo de sistemas normalizados de información de enfermería. Washington (Estados Unidos da América): OPS; 2001.

8. Brandt K. Poor quality or poor design? A review of the literature on the quality of documentation within the electronic medical record (Paper presentation). Comput Inform Nurs. 2008; 26(5):302-3.

9. Bittar DB, Pereira LV, Lemos RCA. Sistematização da assistência de enfermagem ao paciente crítico: proposta de instrumento de coleta de dados. Texto contextoenferm. 2006; 15(4): 617-28.

10. Clark J, Lang N. Nursing's next advance: an international classification for nursing practice. J Am Med Inform Assoc. 1998; 5(4): 335-6.

11. Conselho Internacional de Enfermagem. Classificação Internacional das Práticas de Enfermagem: versão 1.0. Marin HF, translator. São Paulo: Algol; 2007. 12. Matney S, Dlugos M, Haskell R, Saba VA. Translation and integration of CCC nursing diagnoses into ICNP. J Am Med Inform Assoc. 2008; 15(6): 791-3.

13. Murphy CA, Merriman K, Zabka C, Penick M, Villamayor P. Patient-entered electronic healthcare records with electronic medical record integration: lessons learned from the field (Paper Presentation). Comput Inform Nurs. 2008; 26(5): 302.

14. Trentini M, Paim L. Pesquisa convergenteassistencial. 2nd ed. Florianópolis: Insular; 2004.

15. Freire P. Pedagogia do oprimido. 41st ed. Rio de J aneiro: Paz e Terra; 2005.

16. Bordenave JD, Pereira AM. Estratégias de ensino aprendizagem. 12st ed. Petrópolis: Vozes; 1991.

17. International Organization for Standardization. ISO 9241-11: Guidance on Usability (1998) [Internet]. London (UK): UsabilityNet; 2006 Jan 2 [update 2006 J an 2; cited 2009 Agu 25]. Available from: http://www.usabilitynet.org/tools/r_international.htm\#9 241-11.

18. Barra DCC. Processo de enfermagem informatizado em terapia intensiva em ambiente PDA (Personal Digital Assistant) a partir da CIPE ${ }^{\circledR}$ versão 1.0 [dissertation]. Florianópolis: Programa de Pós-Graduação em Enfermagem/UFSC; 2008. $151 \mathrm{p}$.

19. Nóbrega MML, Garcia TR, Araruna JF, Nunes WCAN, Dias GKG, Beserra PFJ. Mapeamento de termos atribuídos aos fenômenos de enfermagem nos registros dos componentes da equipe de enfermagem. Rev. Eletr. Enf. [Internet]. 2003 [cited 2009 apr 18];5(2):33-44. Available

from: http://www.fen.ufg.br/revista/revista5_2/pdf/mapa.pdf 20. Kaplan B. Evaluating informatics applications-some alternative approaches: theory, social interactionism, and call for methodological pluralism. Int J Med Inform. $2001 ; 64(1)$ :39- 56 .

Artigo recebido em 01.04.08.

Aprovado para publicação em 06.05.09.

Artigo publicado em 30.09.09. 